\title{
A Review of Tomography System
}

\author{
Juliza Jamaludina, Mohd Zikrillah Zawahira, Ruzairi Abdul Rahimaª, Fazlul Rahman Mohd Yunusa, Nor Muzakkir Nor Ayoba , Muhammad, \\ Suzzana Ridzuan Awª, Naizatul Shima Fadzila ${ }^{a}$ Zulkarnay Zakariab, Mohd Hafiz Fazalul Rahiman ${ }^{b}$
}

aProcess Tomography and Instrumentation Engineering Research Group (PROTOM-i), Infocomm Research Alliance, Faculty of Electrical Engineering, Universiti Teknologi Malaysia, 81310 UTM Johor Bahru, Johor, Malaysia

${ }^{b}$ Tomography Imaging Research Group, School of Mechatronic Engineering, Universiti Malaysia Perlis, 02600, Arau, Perlis, Malaysia

*Corresponding author: ruzairi@fke.utm.my

\section{Article history}

Received :30 April 2013

Received in revised form : 9 July 2013

Accepted :5 August 2013

\begin{abstract}
Tomography system is a method that being used to reconstruct a cross sectional image in vessel or pipeline. This method is widely used in medical and industrial fields. The aim of this paper is to provide an overview of tomography system based on its basics construction, types of sensors, its applications in today's industry, and image reconstruction. This paper review consists of literature citations which related with tomography system.
\end{abstract}

Keywords: Tomography; sensors; applications; image reconstructions

(C) 2013 Penerbit UTM Press. All rights reserved.

\subsection{INTRODUCTION}

Tomography system is one of the methods that have been used to construct cross-sectional image from data obtained by the system. [1] Basics concept of tomography is to determine the distributions of materials by obtaining the data using sensors that distributed around the periphery of pipeline. [11] Tomographic imaging system designed to analyze the structure and compositions of objects by examining them with waves or radiation and by calculating virtual cross sections through them. [5] This system also helps us to visualize the internal characteristics such as to measure the concentration, velocity, mass flow rate, and particle sizes distribution. [11]

Tomography method is widely used in medical application before it spread into industrial application. Impedance tomography is one of the tomography methods that applied in medical field. This method is imaging the distribution of conductivity or permittivity volume inside human body. Impedance tomography is used in medical area because this method is safe, simple application, high speed data collection and has possibility to characterized tissue and other particles in human body. [30] Another approach of medical tomography system is by using optical coherence tomography. Optical coherence tomography technique is non invasive and non intrusive technique where this method can generate two dimensional and three dimensional tomographics images with micron resolution. [46]

Tomography method also applied in industry and it is known as process tomography. Process tomography mostly used in mixing process, multiphase flow, batch process monitoring, and many more. [11] Impedance tomography system also had been used in process tomography to detect particles in flow measurement system. The types of sensor still the same but the orientation of sensors is difference. Some of process tomography system is developed in using wireless local area network for data processing. This wireless system had help to reduce the noise effect that can cause low quality of image reconstruction. [30] Process tomography system should be robust and can perform well in aggressive environment which contains flammable superheated or corrosive materials and high internal pressure. [35]

\subsection{HISTORY OF TOMOGRAPHY SYSTEM}

Tomography system already started at 1950 in medical field. In 1990 , process tomography becomes important part in industry. At that moment, the requirement of instrument that robust, non invasive, and can operate with fast moving fluid is highly increased. [2] In the middle of 1980, electrical impedance tomography is develop for imaging human body because it safe and low cost. The difference between medical tomography and process tomography is the type of object that the system measured. For medical tomography, it aims to measure the locations of objects in space where process tomography needs to measure both locations and velocity of movements. [2] Medical tomography and process tomography also produce different quality of image reconstruction. For medical tomography, it gives good image reconstruction compare to process tomography. It is because process tomography deal with high level of noise. [12] Besides that, tomographic reconstruction in process applications has been more quantitative than qualitative in real world. In order to minimize this problem, some researchers had developed and applied process tomography system as a sensor for optimal 
control. In this case, they applied feedback control such as proportional-integral controller, proportional -integralderivative controller and linear quadratic Gaussian controller with tomography system. [15]

They are four generation of tomography system. First generation consists of source which emits a linear beam radiation sources. While in second generation, the detectors is placed opposites to a set of radioactive sources, sometimes the sensors moving around the objects measured, and numbers of receivers is equal to the numbers of transmitter. For third generations, various types of detectors being used and fan beam projection is introduced. [35] The last generation is called the fourth generation where tomography system should be fixed and no need to move their transmitter and receiver around the objects. [36]

\subsection{BASIC CONSTRUCTION OF TOMOGRAPHY SYSTEM}

The development of tomography system divides into three segments; sensors, data acquisition system, and software. [6] Tomography system has two main components which is hardware part and software part. For hardware its contain sensors, signal or data control, while software contain signal reconstruction display. [1] The right choice of sensors is needed because sensors is the main part in tomography system and it is chosen based on the types of particles or objects that we want to measure whether it is liquid, gas, or multiphase mixture. [1] Sensors perform indirect measurement of the image by detecting the radiation with which the object examined. [5] The signals from sensor usually being amplified, filtered, and multiplex before the data is sent to the computer. [22] There are two types of sensors, hard-field sensor and soft- field sensors. For hard-field sensors, the sensitivity is independent of parameter distribution inside the sensor while for soft-field sensors; the sensitivity distribution inside the fields depends on the parameter distributions. [22] An example of hard field tomography systems are gamma tomography method and X-Ray tomography method. These two methods are so expensive and required special attention of safety. Electrical tomography system is an example for soft field method. [49]

Different types of tomography consist of different type of sensors being used. One type of tomography system which is ionizing radiation can gives high resolution image. X-ray computed tomography system is suitable for off-line process because it is bulky while gamma radiation is one method for real time process tomography. It is suitable to used optical tomography system to measure transparent liquid or fluid. Electrical resistance tomography is suitable for process that does not have electrically insulating boundary while electrical capacitance was inversed. [1] Electrical capacitance tomography system has three difference subsystems; DC offset compensation, AC feedback compensation, and self balancing. All these three subsystem have their owned advantages and disadvantages.[29] Tomography system that used capacitance, resistance, and inductance are known as electrical tomography system and these electrical tomography system is non intrusive and low cost, no radiation hazards and easy to implement.[3] Besides that, electrical tomography system is rugged and robust to cope with industrial environment.[28] This type of tomography system also suitable for real time imaging system because it have high speed capability.[28] To measure the density properties of distributed phases, ultrasonic tomography is the right choice. [1]

Sensor configuration also important parts to be investigate. There are many types of projection patterns that being used in tomography field to detect the flow materials within a pipeline. In optical tomography, there are two types of projection beams, parallel beam projections and fan beam projections. For parallel beam projections, the sensors are in a straight line and the view angle for each should be small enough. $[25,51]$

\subsection{APPLICATION OF TOMOGRAPHY SYSTEM}

Many industries such as power generation, food processing, paper and plastics productions, and solid waste treatment deal with gas or solids flow measurement. [13] Process tomography system is useful for plant control because the ability of this system to measure concentration, identify phase size and boundaries within vessels and pipeline. This technique is non invasive system and non intrusive system where it is not applied directly to the object that want to measure and the nature of object also not being touched. [2] Process tomography involved in two different types of systems which are on line and off line systems. For on line system, data captured should be fast and data processing time also should be fast while for offline system data captured should be fast but data processing time can be slow. [3]

Process tomography system can be found in single modality system and multi modality system. Single modality system means only one type of sensors involved in measurement. This system cannot work to measure multiphase flow because it will produce low quality of image reconstruction. For multiphase flow, multi modality tomography system is introduced. Unfortunately, multi modal tomography system has certain requirement to make it work smoothly. The synchronization in two modality data acquisition is required. Besides that, multi modal tomography system should be fast, standardized, flexible to adapt the difference processes, and can integrate into one measurement system. This system also need good software because it acts as main controller to control and communicates with each modal unit via communication link. This software tools also play an important part to achieve central system management for multi modal system with advanced data processing and visualization. [24] Multi modal tomography systems help to increase the speed of monitoring image, and this approach is really needed by today's industry for optimization and troubleshooting requirement. [35] Multi modal tomography system usually used in chemical and biological process industry that used multiphase flow systems and the flow structure is complicated because it involved micro-scale and macro-scale. [50]

\subsection{Tomography System In Chemical Engineering}

Most of process tomography system is used for online control and monitoring purpose because of its capability to produce data for internal characteristics of chemical engineering process. [3] This process tomography system widely used in chemical engineering process such as bubbles columns, fluidized beds, pneumatic transport, liquid mixing, cyclonic separation, pressure filtration, liquid pipe flow and many more. [3] Most of the used of tomography system in chemical engineering is for qualitative and quantitative analysis and fault detection process where this method can produce three dimensional visualization in real time process. [6]

Process tomography also had been used in oil industry for multi phase flow measurement system. Oil industry faced with difficulty to measured complex mixture of gas, oil, water and other components such as sand. [7] Besides that, oil reservoirs were totally different compare to each other because it based on the location and the edge of wells. [10] Conventional approach that usually used to measure this complex of mixture oil is using 
single phase flow meter where the mixture need to separate based on it characteristic by using separator. The problems occurred because some of separators are bulky, high installation cost, and considerable maintenance and some of them have limitations and can only deal with homogenous flow. [7] Oil industries really need the instrument that accurate for each phase, non intrusive, reliable, flow regime independent and suitable for use over the full components fraction range. [10] Process tomography system is the suitable method that can be used in oil industry that deals with complex process. More than one type of process tomography systems can be use to produce an accurate data for image reconstruction. [8] There are varieties of sensors in today's market and each of sensors has its own ability to measured different velocity profile and different phase distribution. [9] Combinations of different process tomography techniques can help multi phase measurement industry to identify various flow patterns and boundaries [9].

Some process industry deal with gas-liquid phase or gasliquid-solid phase. Usually chemical, petrochemical, biochemical, and environmental engineering use bubble column for certain operation which deal with air and liquid such as petroleum refining, hydrogenation, oxidation, crystallization, fermentation, coal liquefaction, wastewater treatment, and air pollution control. [32]

Another application that used tomography system is monitoring carbon dioxide storage. This method is used by monitoring the image reconstruction that produces from sensors applied in the carbon dioxide storage. From the image reconstruction, operator can control the amount of carbon dioxide reaction by monitoring from control room. This is very important part for reducing greenhouse gas emissions from spread into our atmosphere that can pollute our ecosystem. [17]

\subsection{Tomography System In Geophysics}

Tomography also widely used in geophysics field especially for seismic depth imaging. Seismological tomography deals with the problem related to travel time residuals defined as differences between the observed and calculated arrivals time. [31] The huge data captured by seismic tomography contributes inconsistencies and errors in data results from incorrect coordinates, errors in timing acquisitions, and error in merging procedure. [31] The evolution in seismic tomography area has solved many problems that occurred based on this old method of tomography. For an examples, the improvement in seismic tomography have help increased the resolution from few thousands meters to a few hundred meters, strategy of working also change from long wavelength to short wavelength solutions and the image quality also improved. Grid tomography system is very important to monitoring requirement of land and ocean bottom cable. [16]

Local earthquake tomography also becomes the most important application that being used in seismic network. In local earthquake tomography, the accurate exact data of location is needed. Unfortunately, seismic tomography face with many problems related to the accuracy of data such as network geometry, arrival time accuracy and presence of noise. To solve this problem, some researchers have introduced double difference seismic tomography method which deals with new algorithms and calculation related to both absolute and relative arrival time. [21]

Submarines landslides analysis is very important because submarines landslide become one of the factors that cause tsunami and earthquake which give great destroy to population and infrastructures. Due to this case, fluidization process in submarine landslide analysis is very important to study. Computer axial tomography is used in this physical area to study the instability of slide and helps to reconstruct an image of the soil-water interaction. A landslides investigation is very important to monitoring the influence of water circulation on the motion of landslide by producing image visualization. Computer axial tomography is the right choice of sensor for soil science analysis, sediment logy analysis, and coal geology or rock mechanics because it is non destructive technique. [38]

Acoustic tomography is another method that applied for river discharge monitoring because it is the procedure to control water resources. Usually, horizontal acoustic Doppler and acoustics velocity meters are used to measured stream flow. But these methods have disadvantage where only limited number of velocity sample can be distributed in cross section of river stream. [41] Acoustic tomography system work based on ultrasound detection and illumination to reconstruct image from data captured. [40] This tomography method has ability to measure river discharge even under floods, high turbidity concentration, and high noise level. It is because this method is non destructive system. [41]

One of the successful applications of tomography system is being used in an atmospheric science field. The tomography system is known as OSIRIS which is the combination of visible optical spectrograph and infrared imager. The main used of OSIRIS in open satellite is to observe the important process in atmosphere that can cause global warming. [19]

\subsection{Tomography System In Botanical Field}

Many experiment based on the properties of soil, root, leaves, wood are carry on using tomography method. Optical Coherence tomography had been used for investigation of wood finishes. This method has the ability to detect layer thickness and can reconstruct the image of wood below the varnishes. This study is important for investigation related to heritage artifacts. [20]

Electron tomography system had been used more than half century for plant thylakoid membranes analysis. This method is used to investigate the complex structure and organization of chloroplast thylakoid membranes. [44] Tomography method that using ion beam scanning electron microscope is one of the powerful methods for characterization of three dimensional of micro and nanostructures. [48]X-Ray micro computed tomography is use for visualizing plant leaf structures. In early edition of plants leaf structures analysis, researchers used confocal microscopy. But this method has limitation where it only can view limited size area and high transparency of leaf. Sometimes, they need high refractive staining agents to enhance the contrast of leaf. By using X-Ray micro computed tomography system, it can give precise of image visualization and right quantification of internal structures in three dimensional. Besides that, the advanced of computer software help to increase the quality of image reconstruction until the characteristics of leaf such as the porosity, connectivity and surface area can visualize in three dimensional. [39] X-Ray computed tomography system also can be used for root-soil interaction study. This is because this method is nonintrusive so the components of the roots and soil are not disturbed. X-Ray computed tomography method also provide the image of nature root growth in different types of soils. This study helps to improve the development of botanical sector. [43] For soil textures image reconstruction, electrical resistive tomography is the suitable method. This method is capable to detect stones, tillage effects in soil, soil texture, bulk density and water contents. It is important part for fertilization of soil check up. [42]

Industrial pulp mixer use electrical resistance tomography to check the mixing quality and quantity in vessel pipeline. Industrial that deal with pulp mixer really needs tomography 
method to control the quality product in controlling consistency of pulp processing. If good mixing cannot be control, surely operation costs can be higher due to unstable production and poor product quality. The way that tomography method controls the mixer is by given an image reconstruction of mixing product. From the image captured, operator can monitor the quality of the mixing pulp by analysis the concentration of the image data. For an example, image reconstruction that show the wide area of lower conductivity indicates higher concentration of chlorine dioxide while small regions of low conductivity show lower concentration of chlorine dioxide. [45]

\subsection{Tomography System In Powders Technology}

The development of powder technologist also based on the important of process tomography system where this method becomes the important tools for monitoring particulate structures and moving particulates structure. [14]Electrodynamics tomography system had proved its capability to captured velocity and mass flow rate profiles of dry powders in a gravity drop conveyer. To measure the velocity of particles is based on the transits times of particles from upstream of electrodynamics sensors to downstream of electrodynamics sensors. [27] Tomography system also used for structure characterization of metallic ceramic composite foam. In this case, computer tomography system is used to examine the composite of foam, porous structure and analyze the geometry structure of the foam. [34]

\subsection{High Voltage Tomography System}

Based on the improvement of study in tomography area, new development which is known as high voltage electrical capacitance tomography had being introduced. This is one of the success achievements in tomography research where tomography system can be applied for large equipment. Process reaction that happened in large equipments generally changes very slowly and faced with huge noise from many possibilities. So, high voltage electrical capacitance tomography was developed by researchers to deal with this problem. Usually the new development always need some improvement where the image reconstruction from this new invention still facing with low quality of resolution. [37]

\subsection{IMAGE RECONSTRUCTION}

Image reconstruction is mean to combine the data obtained from sensors that captured image of object measured at different angles and views. [26] To obtained final images, appropriate algorithms are applied to solve equation and reconstruct virtual cross section. [5] There are three methods for image reconstruction, linear methods, iterative methods, and heuristic methods. Linear method is the simplest and quick method compares to the others methods. Linear back projection is widely used in linear method but this method produce poor image reconstruction for analysis and this approach is used in early stage of medical tomography. [22] Linear back projection produce blurry image and hard to captured scattered images. Usually, filtering is applied in this algorithm to enhance the image reconstruction. This common algorithm is known as filtered back projection algorithm. [26] For non linear iterative methods, it is not suitable for real time image reconstructions because the computational load makes them too slow to process the data. But, this method is easy to model and can handle projection noise. [22] Heuristic method can be linear or non linear. This method is based on the relationship of trained images and measurements images. [4] Image analysis mostly based on pixels value. Simple image analysis base on mean, minimum, or maximum of pixels values. These pixels values give information related to concentration, filling fraction, or packing density. [4]

Reconstruction technique for tomography system is known as inverse problem. [22] Inverse problem can divide into two different part, estimation components and appraisal components. For estimation component, the reconstruction image is based on data received. While for appraisal components, it based on the relationship between estimated model and actual model. [18]

Some researchers state that, to improve the quality of image reconstruction, the numbers of sensors should be increased. [22] Another approach to improve the quality of image reconstruction is by improving the reconstruction algorithm so that more information could be extracted from existing data. [22] New development such as neural networks and binary optimizer should be investigated for the use of image reconstruction. [23] Some sources said that the low quality of image reconstruction is because of the limitation of radial and angular sampling of image sliced. In a way to conquered this problem, sinusoidal Hough transform was created by researchers. [33] But, there are still limitation occurred cause by another part such as computational cost, execution time, and limitation in real time capability. [33]

\subsection{CONCLUSIONS}

Tomography system is widely use in medical in 1950 and in industrial since 1990. The main reason for the development of tomography system is to capture cross sectional image of internal object for analysis and monitoring requirement. Basic construction of tomography system is based on hardware and software part. The suitable type of sensor is needed to produce high quality of image reconstruction. The development of tomography method is synchronous with the development of process industrial. New inventions of tomography system are developing to full fill the requirement of industries that face with many complexities such as multiphase flow measurement and large equipment. Tomography systems are important for other sector such as geophysics, botanical and powders technologist. More research need be studied in a way to solve certain problems occurred and to improve the quality of tomography method and its image reconstruction.

\section{Acknowledgement}

The authors would like to thank the PROTOM research group for helping giving information for this review paper.

\section{References}

[1] M. S. Beck, R. A. William. 1998. Process Tomography-The State of Art. Transducer and Instrumentation. 20(4): 163-177.

[2] M. S. Beck, R. A. William. 1996. Process Tomography: European Innovation and Applications. Measurement Science Technology. 7: 215224.

[3] C. G. Xie, N. Renecke, M. S. Beck, D. Mewes, R. A. Williams. 1995. Electrical Tomography Technique for Process Engineering Applications. The Chemical Engineering Journal. 56: 127-133.

[4] H. S. Topp, A. J. Peyton, E. K. Kermley, R. H. Wilson. 2003. Chemical Engineering-Application of Electrical Process Tomography. Sensors and Actuators. 92: 17-24.

[5] G. Pierre. 2009. Digital Signal and Image Processing-Tomography. John Wiley and Sons Inc. 1: 1-3.

[6] R. A. Williams. 1995. Tomographic Imaging for Modeling and Control of Mineral Process. The Chemical Engineering Journal. 59: 71-85. 
[7] I. Ismail, J. C. Gamio, S. F. A. Bukhari, W. Q. Yang. 2005. Tomography for Multi-Phase Flow Measurement in the Oil Industry .Flow Measurement and Instrumentation.16: 145-155.

[8] B. S. Hoyle , X. Jia, F. J. W. Podd, H. I. Schlaberg, H. S. Tan, M. Wang, R. M. West, R. A. Williams, T. A. York. 2001. Design and Application of A Multi-Modal Process Tomography System. Measurement Science and Technology.12: 1157-1165.

[9] T. Dyakowski. 1996. Process Tomography Applied to Multi-Phase flow Measurement. Measurement Science and Technology. 7: 343-353.

[10] T. Richard, G. A. Johansen, E. A. Hammer. 1999. Three Phase Flow Measurement in the Offshore Oil Industry-Is There A Place for Process Tomography? First World Congress on Industrial Process Tomography Buxton Greater Manchester. 228-235.

[11] T. York. 2001. Status of Electrical Tomography in Industrial Applications. Journal of Electronic Imaging. 10(3): 606-619.

[12] R. M. West, X. Jia, R. A. Williams. 2000. Parametric Modeling in Industrial Process Tomography. Chemical Engineering Journal. 77: 31 36.

[13] W. Q. Yang, S. Liu. 2000. Role of Tomography in Gas/Solids Flow Measurement. Flow Measurements and Instrumentation. 11: 237-244.

[14] R. A. William, X. Jia. 2003. Tomographic Imaging of Particulate Systems. Advanced Powder Technology. 14 (1): 1-16.

[15] A. R. Ruuskanen, A. Seppanen, S. Duncan, E. Somersalo, J. P. Kaipio 2006. Using Process Tomography as a Sensor for Optimal Control. Applied Numerical Mathematics. 56: 37-54.

[16] M. J. Woodward, D. Nichols, O. Zdraveva, P. Whitfield, T. Johns. 2008 A Decade of Tomography. Geophysiscs. 73(5): 5-11.

[17] P. Bergmann, C. S. Hattenberger, D. Kiessling, C. Rucker, T. Labizke, J. Henningses, G. Baunmann, H. Schutt. 2012 .Surface Downhole Electrical Resistivity Tomography Applied to Monitoring of Carbon Dioxide Storage at Ketzin, Germany. Geophysics. 77(6): 253-267.

[18] G. A. Oldenborger, P. S. Routh, M. D. Knoll. 2007. Model Realibility for 3D Electrical Resistivity Tomography: Application of the Volume of Investigation Index to A Time-Lapse Monitoring Experiment. Geophysics.72(4): 167-175.

[19] E. J. Llewellyn, D. A. Degenstein, I. C. McDade, R. L. Gattinger, R. King, R. Buckingham, E. H. Richardson, D. P. Murtagh, W. F. J. Evans, B. H. Solhem, K. Strong, and J. C. McConnell. OSIRISIS-Applications of Tomography for Absorbed Emission in Remote Sensing. Bulletin of the Seismological Society of America. 1-6.

[20] G. Latour, J. P. Echard, B. Soulier, I. Emond, S. Vaiedelich, M. elias 2009. Structural and Optical Properties of Wood And Wood Finished Studied Using Optical Coherence Tomography: Application to An $18^{\text {th }}$ Century Italian Violin. Applied Optics. 48(33): 6485-6491.

[21] H. Zhang, C. H. Thurber. 2003. Double Difference Tomography: Method and Its Application to the Hayward Fault, California. Bulletin of the Seismological Society of America. 93(5): 1875-1889.

[22] C. Michelsen. 1995. A Review of Reconstruction Techniques for Capacitance Tomography. Measurement Science Technology.7: 325337.

[23] N. Reinecke and D.Miewes. 1996. Recent Developments and Industrial/Research Applications of Capacitance Tomography. Measurement Science Technology.7: 233-246.

[24] C. Qiu, B. S. Hoyle, F. J. W. Podd. 2007. Engineering and Application of a Dual Modality Process Tomography System. Flow Measurement and Instrumentation. 18: 247-254.

[25] R. A. Rahim, P. J. Fea, C. K. San, L. L.Chean. 2004. Real Time Image Reconstruction for Various Flow Regimes Using an Optical Tomography Sensor in A Gravity Flow Conveyer. Advanced Powder Technology. 16(4): 399-411.

[26] G. L. Zeng. 2001. Image Reconstruction-A Tutorial. Computerized Medical Imaging and Graphics. 25: 97-103.

[27] R. G. Green, M. F. Rahmat, K. Dutton, K. Evans, A. Goude, and M. Henry. 1997. Velocity and Mass Flow Rate Profiles of Dry Powders in a Gravity Drop Conveyer Using An Electrodynamics Tomography System. Measurement Science Technology. 8: 429-436.

[28] T. Dyakowski, L. F. C. Jeanmeure, A. J. Jaworski. 2000. Application of Electrical Tomography for Gas, Solids, and Liquid Solids Flows. Powder Technology. 112: 174-192.

[29] W. Q. Yang. 1996. Hardware Design of Electrical Capacitance Tomography Systems. Measurement Science Technology. 7: 225-232.

[30] B. H. Brown. 2001. Medical Impedance Tomography and Process Impedance Tomography: A Brief Review. Measurement Science Technology. 12: 991-996.

[31] V. Maurer, E. Kissling, S. Husen, and R. Quintero. 2010. Detection of Systematic Errors in Travel Time Data Using a Minimum ID Model: Application to Costa Rica Seismic Tomography. Bulletin of the Seismological Society of America. 100: 629-639.
[32] J. Haibo, L. Yieching, Y. Suohe, G. He, Z. Guo. 2012. The Parameters Measurement of Air Water Two Phase Flow Using Electrical Resistance Tomography (ERT) Technique in A Bubble Column. Flow Measurement and Instrumentation.

[33] J. A. C. Ceballos. 2013. Parallel Data Reconstruction for Limited Views Tomography Sensors by Sinusoidal Hough Transform. IEEE Sensors Journal. 13(2): 581-588.

[34] J. Grabian, K. Gawdzinska, L. Wojnar, W. Przetakiewicz. 2013. An Application of Computer Tomography for Structure Characterization of Metallic Ceramic Composite Form. Solid State Phenomena. 197: 226231.

[35] C. H. Mesquita, W. A. Calvo, D. V. Souza, F. E. Sprenger, P. A. V. Salvador, F. E. Costa, M. M. Hamada. 2012. Development of the Mechanical System on a Third Generation Industrial Computed Tomography Scanner in Brazil. Journal of Physical Science and Application. 2(6): 158-165.

[36] C. H. Mesquita, C. R. Dantas, F. E. Costa, D. V. S Calvalho, M. M. Hamada. 2010. Development of a Fourth Generation Industrial Tomography for Multiphase System Analysis. Journal of Physical Science and Application.

[37] L. Decai, W. Jixiang, S. Fuqun. 2013. The Research of High Voltage Electrical Capacitance Tomography System. Applied Mechanics and Material. 303: 896-899.

[38] S. W. Jeong, J. Locat, S. Lerouiel, J. L. Robert. 2013. Fluidization Process in Submarine Landslides: Physical and Numerical Considerations.Marine Georesources and Geotechnology. 31: 190-207.

[39] R. Pajor, A. Fleming, C. P. Osborne, S. A. Rolfe, C. J. Sturrock, S. J. Mooney. 2013. Seeing Space Visualisations and Quantifications of Plant Leaf Structure using X-Ray Micro Computed Tomography. Journal of Experimental Botany. 64(2): 385-390.

[40] J. Provost, F. Lesage. 2009. The Application of Compressed Sensing for Photo Acoustic Tomography. Transaction on Medical Imaging. 28(4): 585-594.

[41] K. Kawanisi, M. Razaz, A. Kaneko, S. Watanabe. 2010. Long Term Measurement of Stream Flow and Salinity in a Tidal River by the Use of the Fluvial Acoustic Tomography System. Journal of Hydrology. 380: 74-81.

[42] R. Rosst, M. Amato, A. Pollice, G. Bitella, J. J. Gomez, R. Bochiccio, S. Baronti. 2013. Electrical Resistivity Tomography to Detect the Effect of Tillage in a Soil with a Variable Rock Fragment Content. European Journal of Soil Science. 1-10.

[43] S. Tracy, C. Black, J. Roberts, S. Mooney. 2010. Visualizing the Effect of Compaction on Root Architecture in Soil Using X-Ray Computed Tomography. World Congress of Soil Science; Soil Solution for a Changing World. 160-163.

[44] B. Daum, W. Kuhlbrandt. 2011. Electron Tomography of Plant Thylakoid Membranes. Journal of Experimental. 62(7): 2393-2402.

[45] W. Yenjaichon, G. Pageau, M. Bhole, C. P. J. Bennington, J. R. Grace. 2011. Assessment of Mixing Quality for an Industrial Pulp Mixer Using Electrical Resistance Tomography. The Canadian Journal of Chemical Engineering. 89: 996-1004.

[46] U. Marx, D. Pikert, A. Heymer, R. Schmitt. 2013. Non Invasive Quality Control for Production Process of Artificial Skin Equivalent by Optical Coherent Tomography. The First CIRP Conference on Bio Manufacturing. 5: 128-132.

[47] A. J. Wu, K. A. Goodman. 2013. Positron Emission Tomography Imaging for Gastroesophageal Junction Tumours. Seminar in Radiation Oncology. 10-15.

[48] D. A. M. Winter, C. T. W. M. Schneijdenberg, M. N. Lebink, A. J. Verkleij, M. R. Drury, B. M. Humbel. 2008. Tomography of Insulating Biological and Geological Material Using Focused Ion Beam (FIB) Sectioning and Low Kv BSE Imaging. Journal of Microscopy. 233(3): $372-383$.

[49] M. A. Bennett, R. M. West, S. P. Luke, R. A. William. 2002. The Investigation of Bubble Column and Foam Process Using Electrical Capacitance Tomography. Minerals Engineering. 15: 225-234.

[50] W. A. Al-Masry, E. M. Ali, S. A. Alshebeilli, F. M. Mousa. 2010. Non Invasive Imaging of Shallow Bubbles Columns Using Electrical Capacitance Tomography. Journal of Saudi Chemical Society. 14: 269280.

[51] R. Abdul Rahim. S. Z Mohd. Muji (January 2013). Optical Tomography: Image Improvement using Mix Projection of Parallel and Fan Beam Mode. Measurement Journal (ISSN: 0263-2241) Elsevier Science. No. 46: $1970-1978$ 